\title{
Culture-based antibiotic susceptibility testing for Helicobacter pylori infection: a systematic review
}

\author{
Vincenzo De Francesco ${ }^{a}$, Angelo Zullob, Raffaele Mantac, Alissa Satriano ${ }^{d}$, Giulia Fiorinie, \\ Matteo Pavonie, Ilaria M. Saracino ${ }^{e}$, Fabrizio Giostraf', Giorgio Montif, Dino Vairae
}

Riuniti Hospital, Foggia; Nuovo Regina Margherita Hospital, Rome; Generale Hospital, Perugia; Villa Aurora Clinic, Foligno, Perugia; University of Bologne; Sant'Orsola Hospital, Bologna, Italy

\begin{abstract}
Background Primary antibiotic resistance in Helicobacter pylori (H. pylori) strains is increasing worldwide, affecting therapy success. The use of therapies tailored on susceptibility pre-testing at culture has been proposed, but data are still conflicting.

Method We performed a systematic review to evaluate the role of a culture-based therapeutic approach for $H$. pylori treatment, taking into account the sensitivity of culture and the success rates achieved with tailored therapies in different therapeutic steps.

Results We analyzed data from 51 studies. Overall, $H$. pylori strains were isolated in $80.7 \%$ of 7889 patients, the success rates being $78.1 \%, 77.5 \%, 86.3 \%$ and $86.6 \%$, before first-, second-, third-line or more therapies, respectively. In comparative studies, the infection was cured in $89.9 \%$ of 2052 patients treated with tailored therapies, and in $77.6 \%$ of 2516 patients receiving empiric therapy $(\mathrm{P}<0.001)$. However, in the subanalysis, the tailored approach achieved optimal eradication rates $(>90 \%)$ only when it was applied before first- and second-line therapies, but not before third-line or more attempts $(<80 \%)$. Moreover, no significant difference emerged between the 2 approaches when data from only the most recent (last 5 years) studies were considered, as well as in those performed in Western populations.

Conclusions The attempt to achieve antibiotic susceptibility testing before treatment failed in $20 \%$ of infected patients managed in dedicated laboratories. Culture-tailored therapies administered after 2 or more therapies achieved suboptimal eradication rates. The role of bacterial culture in patients whose therapeutic management failed to eradicate $H$. pylori probably needs to be corroborated by further data.
\end{abstract}

Keywords Helicobacter pylori, culture, therapy, resistance

Ann Gastroenterol 2022; 35 (2): 127-134

a'Gastroenterology Unit, "Riuniti” Hospital, Foggia (Vincenzo De Francesco); 'Gastroenterology and Digestive Endoscopy, "Nuovo Regina Margherita" Hospital, Rome (Angelo Zullo); 'Gastroenterology and Digestive Endoscopy, "Generale Hospital", Perugia (Raffaele Manta); ${ }^{\mathrm{d} C l i n i c a l}$ Nutrition Unit, "Villa Aurora" Clinic, Foligno, Perugia (Alissa Satriano); ${ }^{\mathrm{e}}$ Department of Medical and Surgical Sciences, University of Bologna (Giulia Fiorini, Matteo Pavoni, Ilaria M. Saracino, Dino Vaira);

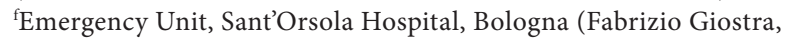
Giorgio Monti), Italy

\section{Conflict of Interest: None}

Correspondence to: Dr. Angelo Zullo, Gastroenterologia ed Endoscopia Digestiva, Ospedale Nuovo Regina Margherita, Via Emilio Morosini, 30, 00153, Rome, Italy, e-mail: angelozullo66@yahoo.it

Received 27 September 2021; accepted 16 December 2021; published online 14 February 2022

DOI: https://doi.org/10.20524/aog.2022.0689

\section{Introduction}

Helicobacter pylori (H. pylori) infection plays a major role in the pathogenesis of different gastroduodenal disorders. These comprise benign diseases, such as non-ulcer dyspepsia, peptic ulcer, idiopathic thrombocytopenic purpura, and idiopathic iron deficiency anemia, as well as tumors, including gastric mucosa-associated lymphoid tissue lymphoma and adenocarcinoma [1-5]. Indeed, $H$. pylori eradication definitely changes the natural history of both peptic ulcer disease and gastric lymphoma, and significantly improves dyspeptic symptoms and platelet count. Moreover, the risk of gastric cancer development is reduced by $44 \%$ following the cure of H. pylori infection [5]. Unfortunately, curing this infection remains challenging for clinicians, as no available empiric therapy regimen is able to achieve bacterial eradication in all treated patients. Therefore, a definite portion of patients 
requires 2 or more treatments in clinical practice. To overcome the impact of antibiotic resistance, administration of therapies tailored on susceptibility pre-testing at culture has been proposed as a more rational approach than empirical use of standard treatment regimens. According to the Kyoto consensus, $H$. pylori gastritis is fundamentally an infectious disease, and principles of antimicrobial stewardship focusing on optimization of therapy to reliably achieve high cure rates and prevent resistance to the antimicrobials should be used [6]. As for other infectious diseases, this approach is expected to achieved bacterial eradication in virtually all patients. Moreover, the diffusion of bacterial resistance toward different antibiotics in other bacterial strains might be limited by reducing the use of empiric therapies. Current Italian and European guidelines suggest that susceptibility pretesting should be performed following 2 or more consecutive therapy failures $[7,8]$. Some studies have even proposed the use of bacterial culture to choose the first-line therapy according to antibiotic susceptibility results $[9,10]$. Nevertheless, clear evidence favoring the tailored over the empiric treatment is still lacking. In addition, isolating H. pylori from gastric biopsies is challenging, and dedicated laboratories for culture, with skilled personnel and specific equipment, are not widespread, limiting the implementation of this approach in clinical practice. Based on these observations, we aimed to evaluate the role of a culture-based therapeutic approach for $H$. pylori treatment by considering the sensitivity of culture and the success rate achieved with tailored therapies in different therapeutic steps.

\section{Materials and methods}

\section{Literature review}

A systematic literature review was performed to find studies reporting data on: a) $H$. pylori culture sensitivity and/or b) the cure rates with successive tailored treatments. Only those studies that provided these data separately according to different therapeutic steps - from before first-line to 3 or more attempts - were considered. Studies that included pediatric patients and those in languages other than English were excluded, and case series with less than 5 patients were not considered. The search was performed in PubMed for publications from January 1, 2004, through March 5, 2021, using the following algorithm (all fields): (Helicobacter pylori OR H. pylori) AND culture. Following title and abstract evaluation, the full text of all relevant studies was retrieved, and the reference lists from the selected articles were reviewed to identify additional studies of potential interest. For each study, the variables extracted were as follows: author, year of publication, country, setting (pre- or posttreatment step), number of patients, the type of culture (E-test or agar dilution) as well as the rate of both successful culture and bacterial eradication. Authors were not contacted in case of absent/incomplete data; those studies were excluded from the analysis. Two investigators (VDF and AZ) extracted data according to a specifically designed database and conflicting data were eventually resolved by another investigator (DV).

\section{Statistical analysis}

Eradication rates and their 95\% confidence intervals (CI) on intention-to-treat (ITT) analysis were computed for each subanalysis. Comparison of cure rates was performed using the chi-squared test. Differences were considered significant at a 5\% probability level. Analyses were performed using Statsoft version 7.1 (StatSoft Europe GmbH, 22301 Hamburg, Germany) for Windows 10.

\section{Results}

\section{Descriptive analysis}

A total of 3015 citations were found, of which 51 met the inclusion criteria on evaluation (Fig. 1) [11-61]. Eleven studies originated from Japan, 10 from China, 7 from each of Italy and Taiwan, 5 from Spain, 4 from Korea, 2 studies from each Australia and Poland, and 1 study from each of Portugal, USA and Israel. The main characteristics of the studies are shown in Table 1.

\section{Culture sensitivity}

Data on culture sensitivity separately reported for different lines of treatment were available in 41 studies [11-51]. Overall, $H$. pylori strains were isolated in 6371 (80.7\%, 95\%CI 79.8-81.6) of 7889 infected patients. More specifically, culture was performed using a single antral specimen in 5053 patients and it was positive in 4052 of them $(80.1 \%, 95 \%$ CI $79-81.2)$, and by antral plus gastric body mucosa specimens in 2836 patients, being positive in 2319 cases $(81.7 \%, 95 \%$ CI $80.3-83.1)$, without a significant difference between groups. Bacterial growth was

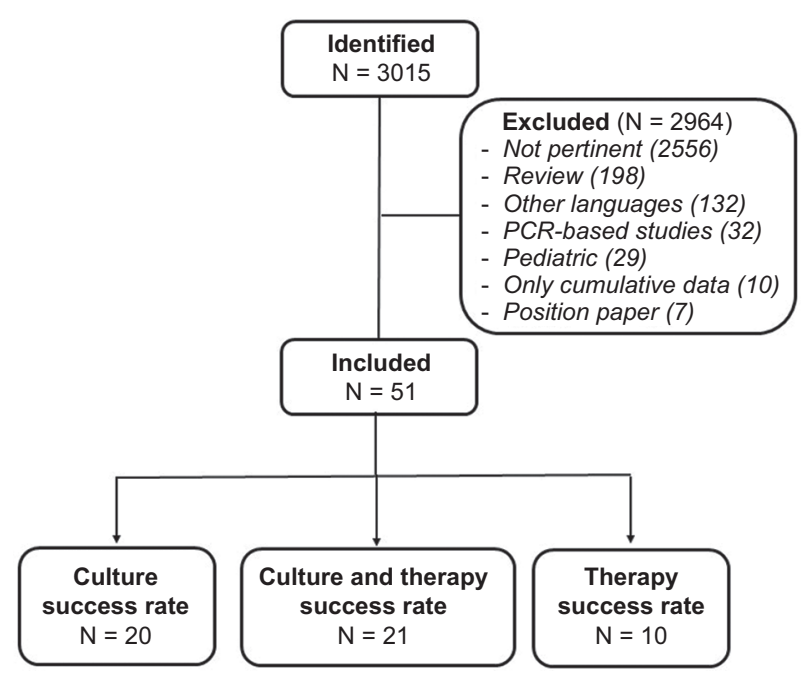

Figure 1 The PRISMA flow diagram $P C R$, polymerase chain reaction 
Table 1 Characteristics of studies

\begin{tabular}{|c|c|c|c|c|}
\hline Ref. & Country & $\begin{array}{l}\text { Patients } \\
\text { (N) }\end{array}$ & Study design & $\begin{array}{l}\text { Therapy } \\
\text { line }\end{array}$ \\
\hline 31 & China & 70 & Prospective & 3 \\
\hline 32 & Italy & 40 & Prospective & 3 \\
\hline 33 & China & 90 & Prospective & 1 \\
\hline 34 & South Korea & 219 & Prospective & 2 \\
\hline 35 & Spain & 104 & Prospective & 1 \\
\hline 36 & Spain & 221 & Retrospective & 1 \\
\hline 37 & Spain & 298 & Prospective & 1 \\
\hline 38 & Italy & $\begin{array}{l}80 \\
83\end{array}$ & $\begin{array}{l}\text { Randomized } \\
\text { Randomized }\end{array}$ & $\begin{array}{l}1 \\
2\end{array}$ \\
\hline 39 & South Korea & 114 & Randomized & 2 \\
\hline 40 & China & 260 & Randomized & 2 \\
\hline 41 & China & 813 & Randomized & 2 \\
\hline 42 & China & 233 & Randomized & 2 \\
\hline 43 & Italy & $\begin{array}{l}415 \\
310 \\
312\end{array}$ & $\begin{array}{l}\text { Retrospective } \\
\text { Retrospective } \\
\text { Retrospective }\end{array}$ & $\begin{array}{c}2 \\
>3\end{array}$ \\
\hline 44 & China & 200 & Prospective & 3 \\
\hline 45 & Poland & 58 & Prospective & 3 \\
\hline 46 & USA & 27 & Retrospective & 3 \\
\hline 47 & Italy & 94 & Prospective & 3 \\
\hline 48 & Italy & 236 & Prospective & 3 \\
\hline 49 & Australia & 279 & Prospective & 3 \\
\hline 50 & Japan & 22 & Randomized & 2 \\
\hline 51 & Japan & 53 & Prospective & 2 \\
\hline 52 & Israel & 98 & Prospective & 3 \\
\hline 53 & Spain & 297 & Prospective & 1 \\
\hline 54 & Spain & 88 & Prospective & 1 \\
\hline 55 & China & 356 & Randomized & 3 \\
\hline 56 & China & 316 & Randomized & 1 \\
\hline 57 & Poland & 120 & Prospective & 1 \\
\hline 58 & China & 668 & Randomized & 1 \\
\hline 59 & South Korea & 146 & Retrospective & 1 \\
\hline 60 & South Korea & 74 & Prospective & 1 \\
\hline 61 & Portugal & 42 & Retrospective & 3 \\
\hline
\end{tabular}

achieved in 2055 (78.1\%, 95\%CI 76.5-79.7) of 2538 patients before first-line therapy, in 2691 (77.5\%, 95\%CI 76.1-78.9) of 3470 patients before second-line, in 1313 (86.3\%, 95\%CI $84.6-88)$ of 1521 patients before third-line, and in 312 (86.6\%, 95\%CI 83.1-90.1) of 360 patients following more therapeutic failures. The success rates of culture performed following either $2(86.3 \%)$ or more $(86.6 \%)$ treatments were significantly higher than those achieved before $(78.1 \%)$ and after $(77.5 \%)$ the first-line therapy $(\mathrm{P}<0.001)$.

\section{Eradication rates}

A total of 31 studies reported data on therapeutic outcomes [31-61]. Of these, 19 studies compared the cure rates between culture-tailored and empiric therapies [31-42,52-58], whilst only the cure rates of tailored therapies were reported in the remaining 12 studies [43-51,59-61].

In comparative studies, the overall cure rate following tailored therapies (1842 of 2052 patients; $89.7 \%$, 95\%CI 88.491.2) was higher $(\mathrm{P}<0.001)$ than that of empiric treatments (1954 of 2516 patients; $77.6 \%, 95 \%$ CI 76-79.2). Specifically, the eradication rates were $91.6 \%(914 / 997)$ and $78.2 \%$ $(1006 / 1285)$, respectively, before first-line therapy $(\mathrm{P}<0.001)$, 91.2\% (702/769) and 79\% (753/953) $(\mathrm{P}<0.001)$ before secondline, and $79 \%(226 / 286)$ and $70.1 \%(195 / 278)(\mathrm{P}=0.03)$ before third-line (or more) approaches (Table 2). When subanalyses of these data were performed according to either different periods or diverse geographic areas, some differences emerged. Indeed, a comparison of the results of studies published in the last 5 years with earlier studies showed that tailored therapies administered before third-line treatments were significantly more effective than empiric therapy only in the older, but not in the more recent studies (Table 3). Considering the different populations, the overall $H$. pylori cure rates following tailored and empiric therapy were, respectively, $84.3 \%(2115 / 2491)$ and $79.1 \%(563 / 711)(\mathrm{P}<0.001)$ in Western and $89.2 \%(1632 / 1828)$ and $77 \%(1391 / 1805)(\mathrm{P}<0.001)$ in Asian countries. The success rate of tailored therapies in Western populations was lower than that of Asian patients ( $84.3 \%$ vs. $89.2 \%$; $<<0.001$ ), whilst no difference emerged between empiric therapies $(79.1 \%$ vs. $77 \% ; \mathrm{P}=0.25)$.

Data from 12 non-comparative studies showed a cumulative eradication rate of $83.1 \%(1885 / 2268)$, with values of $88.6 \%$ in 220 patients, $82.4 \%$ in 490 , and $82.5 \%$ in 1558 cases, when the culture was performed before first-, second- and third-line (or more) therapies, respectively (Table 4). The cure rate achieved before first-line $(88.6 \%)$ therapy was significantly higher than that following other steps $(82.4 \%$ and $83.8 \%$; $\mathrm{P}=0.04)$. By totaling all available (comparative and non-comparative) studies, the susceptibility-based approach achieved a cure rate of $91.1 \%(1109 / 1217), 87.8 \%(1106 / 1259)$ and $83 \%$ (1532/1844) before first-, second- or third-line (or more) therapies, respectively, with a statistically significant $(\mathrm{P}=0.03)$ trend in the reduction of cure rates among the 3 steps.

\section{Susceptibility-based method}

As far as the method for antibiotic susceptibility testing used in the considered studies is concerned, the E-test was utilized in 19 series with a total of 2935 patients, and the agar dilution method in 12 studies with 1259 cases. In comparative studies, a similar eradication rate was achieved by therapies tailored on agar dilution testing (811 of $905 ; 89.6 \%, 95 \% \mathrm{CI}$ 87.6-91.6) and that based on the E-test (918 of 1021; 89.9\%, 95\%CI 88-91.7). In non-comparative studies, the overall cure rate achieved in the 4 studies (321 of 354 patients) with 
Table 2 Cure rates at intention to treat analysis following culture-tailored and empiric therapies

\begin{tabular}{|c|c|c|c|c|c|}
\hline $\begin{array}{l}\text { Culture-tailored }{ }^{*} \\
\text { [days] }\end{array}$ & $\begin{array}{c}\text { Cure rate } \\
\mathrm{N}(\%)\end{array}$ & $\begin{array}{c}\text { Empiric } \\
\text { [days] }\end{array}$ & $\begin{array}{c}\text { Cure rate } \\
\mathrm{N}(\%)\end{array}$ & P-value & Ref. \\
\hline \multicolumn{6}{|l|}{ Before first-line } \\
\hline E-A-B-C-F-L-M [14] & $41 / 45(91.1)$ & EBAC [14] & $33 / 45(73.3)$ & 0.03 & 33 \\
\hline O-A-C-L-M [10] & $47 / 50(94)$ & $\mathrm{OAC}[10]$ & $36 / 54(66.6)$ & $<0.001$ & 35 \\
\hline O-A-B-C-M-Tet [10] & $103 / 117(88)$ & $\mathrm{OAC}[10]$ & $51 / 104(49)$ & $<0.001$ & 36 \\
\hline O-A-C-Tin [14] & $87 / 89(97.7)$ & OACT [10] & $182 / 209(87)$ & $<0.001$ & 37 \\
\hline R-A-C-L-Tin [10] & $39 / 41(95.1)$ & RAL $[10]$ & $36 / 39(92.3)$ & 0.6 & 38 \\
\hline O-A-C-L-M [10] & $171 / 181(94.4)$ & EACM $[10]$ & $103 / 116(88.7)$ & 0.07 & 53 \\
\hline O-A-C-M [10] & $39 / 43(90.6)$ & Pylera [10] & $43 / 45(95.5)$ & 0.37 & 54 \\
\hline P-A-C-L-M [10] & $64 / 67(95.5)$ & PACM [10] & $47 / 53(88.6)$ & 0.16 & 57 \\
\hline R-A-C-Tin [10] & $282 / 318(88.6)$ & RACTin or RACB [10] & $271 / 350(77.4)$ & $<0.001$ & 58 \\
\hline P-A-C-M [10] & $41 / 46(89.1)$ & PAC [10] or PBMTet [10] or Seq [10] & $204 / 270(75.5)$ & 0.07 & 56 \\
\hline \multicolumn{6}{|l|}{ Before second-line } \\
\hline O-A-C-M [10] & $117 / 125(93.6)$ & $\mathrm{OAC}[10]$ & $107 / 135(79.2)$ & $<0.001$ & 40 \\
\hline E-A-C-L-M-F [14] & $281 / 313(89.7)$ & EAC [14] & $405 / 500(81)$ & $<0.001$ & 41 \\
\hline E-A-B-L-F [10] & $163 / 182(89.5)$ & EBAL [10] & $44 / 51(86.2)$ & 0.51 & 42 \\
\hline R-A-C-L-Tin [10] & $50 / 51(98)$ & RAL $[10]$ & $26 / 32(81.2)$ & 0.007 & 38 \\
\hline P-A-C-M [7] & $54 / 57(94.7)$ & PAC $[7]$ & $41 / 57(71.9)$ & $<0.001$ & 39 \\
\hline E-A-B-M-Tet-Mox [14] & $37 / 41(90.2)$ & EBMTet or EAMox[14] & $130 / 178(73)$ & 0.02 & 34 \\
\hline \multicolumn{6}{|l|}{ Before third-line } \\
\hline O-A-B-C-L-M-Tet [14] & $128 / 164(78)$ & PAL [10] & $142 / 192(73.9)$ & 0.36 & 55 \\
\hline E-A-C-L-M-Tet [14] & $21 / 30(70)$ & EBMTet [14] & $8 / 10(80)$ & 0.53 & 32 \\
\hline O-A-B-C-M-Tet [14] & $42 / 49(85.7)$ & OBMTet [10] & $31 / 49(63.2)$ & 0.01 & 52 \\
\hline E-A-T-M-L [14] & $35 / 43(81.4)$ & EAMTet [14] & $14 / 27(51.8)$ & 0.008 & 31 \\
\hline
\end{tabular}

Combinations of 2 or more of the cited drugs

$O$, omeprazole; P, pantoprazole; E, esomeprazole; $R$, rabeprazole; A, amoxicillin; B, bismuth; C, clarithromycin; F, furazolidone; L, levofloxacin; M, metronidazole; Mox, moxifloxacin; Tet, tetracycline; Tin, tinidazole; Seq, sequential therapy; Pylera, 3-in-1 capsules containing bismuth subcitrate potassium (140 mg), metronidazole $(125 \mathrm{mg})$, and tetracycline $(125 \mathrm{mg})$

Table 3 Eradication rates following tailored and empiric therapy in different periods

\begin{tabular}{|c|c|c|c|c|c|c|}
\hline \multirow[t]{2}{*}{ Therapy step } & \multicolumn{2}{|c|}{ Period $<2016$} & \multirow[t]{2}{*}{ P-value } & \multicolumn{2}{|c|}{ Period $\geq 2016$} & \multirow[t]{2}{*}{ P-value } \\
\hline & Tailored & Empiric & & Tailored & Empiric & \\
\hline $\begin{array}{l}\text { Before first } \\
\mathrm{N}(\%)\end{array}$ & $317 / 342(92.6)$ & $338 / 447(75.6)$ & $<0.001$ & $597 / 655(91.1)$ & $668 / 834(80.1)$ & $<0.001$ \\
\hline $\begin{array}{l}\text { Before second } \\
\mathrm{N}(\%)\end{array}$ & $502 / 546(91.9)$ & $579 / 724(79.9)$ & $<0.001$ & $200 / 223(89.6)$ & $174 / 229(75.9)$ & $<0.001$ \\
\hline $\begin{array}{l}\text { Before third (or more) } \\
\mathrm{N}(\%)\end{array}$ & $42 / 49(85.7)$ & $31 / 49(63.2)$ & 0.01 & $184 / 237(77.6)$ & $164 / 229(71.6)$ & 0.13 \\
\hline
\end{tabular}

therapies tailored on agar dilution was higher than that of 8 studies (1584 of 1914 patients) based on the E-test results ( $90.6 \%$ vs. $82.7 \%, \mathrm{P}<0.001$ ). By totalling the data of all studies, the success rate was significantly $(\mathrm{P}<0.001)$ higher when therapies were tailored on agar dilution (1132 of 1254; 89.9\%, $95 \%$ CI 88.2-91.6) as compared to the E-test (2502 of 2935 ; $85.2 \%, 95 \%$ CI 84-86.5).

\section{Discussion}

Although several factors are involved, primary and secondary bacterial resistance towards the few available antibiotics are the most relevant for $H$. pylori therapy failure.
More specifically, resistance to clarithromycin, metronidazole and levofloxacin is increasing worldwide and affects the success rate of therapeutic regimens containing these drugs [10]. In contrast, the prevalence of primary resistance towards amoxicillin, tetracycline and rifabutin remains distinctly low $[62,63]$. Therefore, tailoring eradication therapy on the antibiotic susceptibility testing is expected to be successful. We performed this study in order to assess the probability of bacterial recovery at culture and to estimate the success rate of antibiotic susceptibility-tailored therapies in different steps of the therapeutic sequence for $H$. pylori eradication.

Overall, our pooled-data analysis found that the bacterial growth failed in $20 \%$ of infected patients managed in dedicated microbiology laboratories. The data showed that the success rate of culture was significantly higher when performed following 
Table 4 Overall eradication rates at intention to treat following culture-tailored therapies

\begin{tabular}{lcc}
\hline Culture-tailored ${ }^{*}$ [days] & Cure rate N (\%) & Ref \\
\hline Before first-line & $126 / 146(86.3)$ & 59 \\
O-A-B-C-Tet [7 or 10] & $69 / 74(93.2)$ & 60 \\
E-A-B-C-L-Tet [7] & & \\
Before second-line & $335 / 415(80.7)$ & 43 \\
O-A-L-Rif or Seq or Pylera [10] & $18 / 22(81.8)$ & 50 \\
L-A-M-L [7] & $51 / 53(96.2)$ & 51 \\
L-A-C-M [7] & & \\
Before third-line (or more) & $249 / 310(80.3)$ & 43 \\
O-L-A-Rif or Seq or Pylera [10 or 12] & $189 / 200(94.5)$ & 44 \\
E-A-B-C-L-M [14] & $32 / 58(55.1)$ & 45 \\
E-A-C-M [7] & $12 / 27(44.4)$ & 46 \\
E-A-B-M-Tet [10] & $85 / 94(90.4)$ & 47 \\
O-A-B-L-C-Dox [7] & $211 / 236(89.4)$ & 48 \\
O-A-L-Rif [10 or 12] & $265 / 279(94.9)$ & 49 \\
R-A-B-Cip-Rif [10] & $25 / 42(59.5)$ & 61 \\
E-A-B-L-M-Tet [14] & $238 / 312(76.2)$ & 43 \\
O-A-L-Rif or Seq or Pylera [10 or 12] & & \\
${ }^{*}$ Combinations of 2 or more of cited drugs & \\
E, esomeprazole; O, omeprazole; $R$, rabeprazole; A, amoxicillin; B, bismuth; \\
C, clarithromycin; Cip, ciprofloxacin; Dox, doxycycline; L, levofloxacin; \\
M, metronidazole; Rif, rifabutin; Tet, tetracycline; Seq, sequential therapy; \\
Pylera, -in-1 capsules containing bismuth subcitrate potassium (140 mg), \\
metronidazole (125 mg), and tetracycline (125 mg)
\end{tabular}

2 or more therapeutic attempts, compared to that performed in naïve patients or just following first-line therapy, when the value was even lower than $80 \%$. Therefore, the attempt to guide first-line therapy for $H$. pylori failed in 1 of every 5 infected patients. On the other hand, when the culture was attempted in unselected naïve patients who underwent upper endoscopy, the antibiotic susceptibility testing was achieved in only 247 (13.3\%) of 1862 cases [59]. Based on all these observations, tailoring first-line therapy seems to be impractical in clinical practice.

By considering data from more than 4500 patients, we compared the cure rates achieved by therapies tailored on antibiotic susceptibility and those empirically administered. As expected, the culture-tailored therapies were more successful overall than empiric regimens. Moreover, another advantage of the tailored strategy is to achieve bacterial eradication, in keeping with the rules of antibiotic stewardship, whilst empiric therapies inevitably lead to the misuse of antibiotics. Nevertheless, optimal (>90\%) eradication rates were achieved only when the tailored strategy was adopted before first- and second-line regimens, whilst suboptimal $(<80 \%)$ cure rates were observed following 2 or more therapy failures, namely when current European guidelines suggest that culturetailored therapies should be administered [8]. In addition, the therapeutic advantage of culture-tailored first-line therapy was mainly due to the low efficacy of the empiric therapies used as comparator in the studies we evaluated. Indeed, despite the use of first-line regimens including only those antibiotics with a proven susceptibility at culture, the eradication rate was not distinctly higher than $90 \%$. This success rate would appear to be no different to that achieved by the more effective therapies currently available, such as sequential, concomitant and quadruple first-line regimens administered empirically [64]. In addition, the lack of any therapeutic advantage of culturebased therapies clearly emerged in more recent studies, and in Western populations. Some host factors, especially those affecting the proton pump inhibitor metabolism (e.g., CYP 2C19 polymorphisms, etc.) may be involved in the difference between Asian and Caucasian studies.

The failure of an antibiotic-based therapy may depend on several factors, such as drug doses, therapy duration, and potency of the anti-secretory drug used. Moreover, tailored therapy may fail to achieve a $100 \%$ eradication rate because of the impossibility of assembling in this phase a successful therapy regimen that includes only the still available antibiotics to which $H$. pylori is susceptible. Indeed, the synergism among different antibiotics, chosen because of the absence of bacterial resistance, could be lacking. For instance, following the administration of a 14-day bismuth-tetracyclineamoxicillin combination, 3 drugs with no or a very low $(<5 \%)$ primary resistance rate, the eradication rate was as low as $43 \%$ [65]. Therefore, apart from antibiotic susceptibility, drug combinations are a matter for concern in H. pylori. On other hand, the possibility of a hetero-resistant status-i.e., the coexistence of susceptible and resistant $H$. pylori strains at different gastric sites in the same patient-may undermine the correct classification of antibiotic susceptibility in vitro [66]. The same result is expected when antibiotic susceptibility is genetically assessed using a culture-free, polymerase chain reaction-based tool, which overcomes the culture limitation of bacterial growth.

Notably, data from a recent systematic review showed that by adopting an empiric therapeutic sequence with bismuthbased quadruple therapy, levofloxacin- and rifabutin-based regimens following first-line therapy failure, $H$. pylori infection might be cured in virtually all patients, with only 1 in every 170 patients eventually remaining infected [67]. Therefore, the real advantage of resorting to bacterial susceptibility testing before third-line therapy, as suggested in the current guidelines $[7,8]$, probably needs to be corroborated by further data. Finally, our analysis found that the tool used for antibiotic susceptibility testing seem to plays a role in predicting the success rate. Specifically, higher eradication rates were achieved when the therapy was tailored on agar dilution as compared to the E-test. Therefore, the agar dilution method should be preferred when deciding on culture-based antibiotic susceptibility testing, but this method is impractical as it is very laborious and time-consuming.

Some limitations of our study should be acknowledged, such as inclusion of case series with heterogeneous samples (from 20 to 813), the use of an arbitrary timeline, and the absence of any search for publications in other databases. However, we identified as many as 51 studies with more than 4500 patients, so it is highly improbable there are many other studies not present in PubMed able to significantly change the results of this systematic review.

In conclusion, our study found that the probability of isolating $H$. pylori in infected patients by culture in dedicated laboratories is $80 \%$. The overall success rate of tailored 
regimens was higher than that of empiric therapies, but data from more recent studies and those performed in Western population failed to find a statistically significant difference, particularly following 2 or more therapy failures. Even when only antibiotics to which the infection is susceptible were used, the eradication rate was not higher than $90 \%$.

\section{Summary Box}

\section{What is already known:}

- Helicobacter pylori (H. pylori) eradication remains challenging for clinicians, as no available empiric therapy regimen is able to achieve bacterial eradication in all treated patients

- Primary and secondary bacterial resistance towards antibiotics is the most relevant factor for therapy failure

- Culture-based therapeutic approaches are expected to cure the infection in virtually all treated patients

\section{What the new findings are:}

- Data found that H. pylori growth failed in $20 \%$ of infected patients managed in dedicated microbiology laboratories, particularly when performed in naive patients

- Culture-tailored therapies were more successful than empiric regimens, but optimal (>90\%) eradication rates were achieved only when the tailored strategy was adopted before first- and second-line therapies, whilst suboptimal $(<80 \%)$ cure rates were observed following 2 or more therapy failures

- The lack of advantage of culture-based therapies over empiric approaches emerged in more recent studies, and in the Western populations

\section{References}

1. Alakkari A, Zullo A, O'Connor HJ. Helicobacter pylori and nonmalignant diseases. Helicobacter 2011;16(Suppl 1):33-37.

2. Rokkas T, Rokka A, Portincasa P. A systematic review and metaanalysis of the role of Helicobacter pylori eradication in preventing gastric cancer. Ann Gastroenterol 2017;30:414-423.

3. Zullo A, Rago A, Felici S, Licci S, Ridola L, Caravita di Toritto T. Onset and progression of precancerous lesions on gastric mucosa of patients treated for gastric lymphoma. J Gastrointestin Liver Dis 2020;29:27-31.

4. Abrignani MG, Gatta L, Gabrielli D, et al. Gastroprotection in patients on antiplatelet and/or anticoagulant therapy: a position paper of National Association of Hospital Cardiologists (ANMCO) and the Italian Association of Hospital Gastroenterologists and Endoscopists (AIGO). Eur J Intern Med 2021;85:1-13.
5. Elli L, Norsa L, Zullo A, et al. Diagnosis of chronic anaemia in gastrointestinal disorders: A guideline by the Italian Association of Hospital Gastroenterologists and Endoscopists (AIGO) and the Italian Society of Paediatric Gastroenterology Hepatology and Nutrition (SIGENP). Dig Liver Dis 2019;51:471-483.

6. Sugano K, Tack J, Kuipers EJ, et al; faculty members of Kyoto Global Consensus Conference. Kyoto global consensus report on Helicobacter pylori gastritis. Gut 2015;64:1353-1367.

7. Zagari RM, Romano M, Ojetti V, et al. Guidelines for the management of Helicobacter pylori infection in Italy: The III Working Group Consensus Report 2015. Dig Liver Dis 2015;47:903-912.

8. Malfertheiner P, Megraud F, O'Morain CA, et al; European Helicobacter and Microbiota Study Group and Consensus panel. Management of Helicobacter pylori infection-the Maastricht V/ Florence Consensus Report. Gut 2017;66:6-30.

9. López-Góngora S, Puig I, Calvet X, et al. Systematic review and meta-analysis: susceptibility-guided versus empirical antibiotic treatment for Helicobacter pylori infection. J Antimicrob Chemother 2015;70:2447-2455.

10. Megraud F, Bruyndonckx R, Coenen S, et al. European Helicobacter pylori Antimicrobial Susceptibility Testing Working Group. Helicobacter pylori resistance to antibiotics in Europe in 2018 and its relationship to antibiotic consumption in the community. Gut 2021;70:1815-1822.

11. Ueki N, Miyake K, Kusunoki M, et al. Impact of quadruple regimen of clarithromycin added to metronidazole-containing triple therapy against Helicobacter pylori infection following clarithromycincontaining triple-therapy failure. Helicobacter 2009;14:91-99.

12. Borody TJ, Pang G, Wettstein AR, et al. Efficacy and safety of rifabutin-containing 'rescue therapy' for resistant Helicobacter pylori infection. Aliment Pharmacol Ther 2006;23:481-488.

13. Kuo CH, Hsu PI, Kuo FC, et al. Comparison of 10 day bismuth quadruple therapy with high-dose metronidazole or levofloxacin for second-line Helicobacter pylori therapy: a randomized controlled trial. J Antimicrob Chemother 2013;68:222-228.

14. Kuo CH, Hu HM, Kuo FC, et al. Efficacy of levofloxacin-based rescue therapy for Helicobacter pylori infection after standard triple therapy: a randomized controlled trial. $J$ Antimicrob Chemother 2009;63:1017-1024.

15. Liang CM, Tai WC, Hsu PI, et al. Trend of changes in antibiotic resistance in Helicobacter pylori from 2013 to 2019: a multicentre report from Taiwan. Therap Adv Gastroenterol 2020;13:1756284820976990.

16. Chuah SK, Tai WC, Hsu PI, et al. The efficacy of second-line antiHelicobacter pylori therapy using an extended 14-day levofloxacin/ amoxicillin/proton-pump inhibitor treatment-a pilot study. Helicobacter 2012;17:374-381.

17. Dore MP, Tadeu V, Are B, et al. Efficacy of a rescue ciprofloxacinbased regimen for eradication of Helicobacter pylori infection after treatment failures. Gastroenterol Res Pract 2012;2012:484591.

18. Furuta T, Kato M, Sugimoto M, et al; Japan Gast Study Group. Triple therapy with ecabet sodium, amoxicillin and lansoprazole for 2 weeks as the rescue regimen for $H$. pylori infection. Intern Med 2011;50:369-374.

19. Hori K, Miwa H, Matsumoto T. Efficacy of 2-week, secondline Helicobacter pylori eradication therapy using rabeprazole, amoxicillin, and metronidazole for the Japanese population. Helicobacter 2011;16:234-240.

20. Liou JM, Chen CC, Chen MJ, et al. Empirical modified sequential therapy containing levofloxacin and high-dose esomeprazole in second-line therapy for Helicobacter pylori infection: a multicentre clinical trial. J Antimicrob Chemother 2011;66:1847-1852.

21. Murakami K, Okimoto T, Kodama M, Sato R, Watanabe K, Fujioka T. Evaluation of three different proton pump inhibitors 
with amoxicillin and metronidazole in retreatment for Helicobacter pylori infection. J Clin Gastroenterol 2008;42:139-142.

22. Murakami K, Okimoto T, Kodama M, et al. Comparison of amoxicillin-metronidazole plus famotidine or lansoprazole for amoxicillin-clarithromycin-proton pump inhibitor treatment failures for Helicobacter pylori infection. Helicobacter 2006;11:436-440.

23. Murakami K, Sato R, Okimoto T, et al. Maintenance therapy with H2-receptor antagonist until assessment of Helicobacter pylori eradication can reduce recurrence of peptic ulcer after successful eradication of the organism: prospective randomized controlled trial. J Gastroenterol Hepatol 2006;21:1048-1053.

24. Nishizawa T, Suzuki H, Masaoka T, Iwasaki E, Hibi T. A new eradication resistance index as a predictor of metronidazolecontaining second-line treatment of Helicobacter pylori. Digestion 2007;76:215-220.

25. Perna F, Zullo A, Ricci C, Hassan C, Morini S, Vaira D. Levofloxacinbased triple therapy for Helicobacter pylori re-treatment: role of bacterial resistance. Dig Liver Dis 2007;39:1001-1005.

26. Shirai N, Sugimoto M, Kodaira C, et al. Dual therapy with high doses of rabeprazole and amoxicillin versus triple therapy with rabeprazole, amoxicillin, and metronidazole as a rescue regimen for Helicobacter pylori infection after the standard triple therapy. Eur J Clin Pharmacol 2007;63:743-749.

27. Kudo T, Fujinami H, Ando T, et al. Comparison of lafutidine and rabeprazole in 7-day second-line amoxicillin- and metronidazolecontaining triple therapy for Helicobacter pylori: a pilot study. Helicobacter 2012;17:277-281.

28. Togawa J, Inamori M, Fujisawa N, et al. Efficacy of a triple therapy with rabeprazole, amoxicillin, and faropenem as second-line treatment after failure of initial Helicobacter pylori eradication therapy. Hepatogastroenterology 2005;52:645-648.

29. Tai WC, Lee CH, Chiou SS, et al. The clinical and bacteriological factors for optimal levofloxacin-containing triple therapy in secondline Helicobacter pylori eradication. PLoS One 2014;9:e105822.

30. Wu DC, Hsu PI, Chen A, et al. Randomized comparison of two rescue therapies for Helicobacter pylori infection. Eur J Clin Invest 2006;36:803-809.

31. Huang HT, Wang HM, Yang SC, et al. Efficacy of a 14-day quadruple-therapy regimen for third-line Helicobacter pylori eradication. Infect Drug Resist 2018;11:2073-2080.

32. Mascellino MT, Oliva A, De Angelis M, Pontone S, Porowska B. Helicobacter pylori infection: antibiotic resistance and eradication rate in patients with gastritis showing previous treatment failures. New Microbiol 2018;41:306-309.

33. Dong F, Ji D, Huang R, et al. Multiple genetic analysis systembased antibiotic susceptibility testing in Helicobacter pylori and high eradication rate with phenotypic resistance-guided quadruple therapy. Medicine (Baltimore) 2015;94:e2056.

34. Kwon YH, Kim N, Lee JY, et al. Comparison of the efficacy of culture-based tailored therapy for Helicobacter pylori eradication with that of the traditional second-line rescue therapy in Korean patients: a prospective single tertiary center study. Scand $J$ Gastroenterol 2016;51:270-276.

35. Martos M, Bujanda L, Salicio Y, et al. Clarithromycin for first-line treatment of Helicobacter pylori infection after culture in highresistance regions. Eur J Gastroenterol Hepatol 2014;26:1380-1384.

36. Cosme A, Montes M, Martos M, et al. Usefulness of antimicrobial susceptibility in the eradication of Helicobacter pylori. Clin Microbiol Infect 2013;19:379-383.

37. Molina-Infante J, Pazos-Pacheco C, Vinagre-Rodriguez G, et al. Nonbismuth quadruple (concomitant) therapy: empirical and tailored efficacy versus standard triple therapy for clarithromycinsusceptible Helicobacter pylori and versus sequential therapy for clarithromycin-resistant strains. Helicobacter 2012;17:269-276.
38. Marzio L, Coraggio D, Capodicasa S, Grossi L, Cappello G. Role of the preliminary susceptibility testing for initial and after failed therapy of Helicobacter pylori infection with levofloxacin, amoxicillin, and esomeprazole. Helicobacter 2006;11:237-242.

39. Park CS, Lee SM, Park $\mathrm{CH}$, et al. Pretreatment antimicrobial susceptibility-guided vs. clarithromycin-based triple therapy for Helicobacter pylori eradication in a region with high rates of multiple drug resistance. Am J Gastroenterol 2014;109:1595-1602.

40. Zhou J, Huo $\mathrm{H}$, Wu M, Jiang X. Role of drug sensitivity test in the triple therapy for eradication of Helicobacter pylori. Chin J Gastroenterol 2010;15:358-360.

41. Zhuo RP, Chen XP, Wu SZ, Xie JL, Hu SK. Clinical effects of quadruple therapy based on antimicrobial susceptibility testing in treatment of Helicobacter pylori associated upper digestive tract diseases. Shijie Huaren Xiaohua Zazhi 2015;23:196-201.

42. Kong S, Huang KM, Wang J, et al. Efficacy of tailored secondline therapy of Helicobacter pylori eradication in patients with clarithromycin-based treatment failure: a multicenter prospective study. Gut Pathog 2020;12:39.

43. Saracino IM, Pavoni M, Zullo A, et al. Antibiotic resistance and therapy outcome in $H$. pylori eradication failure patients. Antibiotics (Basel) 2020;9:121.

44. Yu L, Luo L, Long X, et al. Susceptibility-guided therapy for Helicobacter pylori infection treatment failures. Therap $A d v$ Gastroenterol 2019;12:1756284819874922.

45. Szadkowski A, Zemlak M, Muszyński J. Effectiveness of Helicobacter pylori eradication established on the basis of examination of antibiotic resistance of the bacteria. Prz Gastroenterol 2018;13:93-98.

46. Tan B, Yang JC, Young CL, et al. Helicobacter pylori antimicrobial susceptibility testing-guided salvage therapy in the USA: a real life experience. Dig Dis Sci 2018;63:437-445.

47. Cammarota G, Martino A, Pirozzi G, et al. High efficacy of 1-week doxycycline- and amoxicillin-based quadruple regimen in a culture-guided, third-line treatment approach for Helicobacter pylori infection. Aliment Pharmacol Ther 2004;19:789-795.

48. Fiorini G, Vakil N, Zullo A, et al. Culture-based selection therapy for patients who did not respond to previous treatment for Helicobacter pylori infection. Clin Gastroenterol Hepatol 2013;11:507-510.

49. Tay CY, Windsor HM, Thirriot F, et al. Helicobacter pylori eradication in Western Australia using novel quadruple therapy combinations. Aliment Pharmacol Ther 2012;36:1076-1083.

50. Matsumoto Y, Miki I, Aoyama N, et al. Levofloxacin- versus metronidazole-based rescue therapy for $H$. pylori infection in Japan. Dig Liver Dis 2005;37:821-825.

51. Shimoyama T, Fukuda S, Mikami T, Fukushi M, Munakata A. Efficacy of metronidazole for the treatment of clarithromycinresistant Helicobacter pylori infection in a Japanese population. J Gastroenterol 2004;39:927-930.

52. Yahav J, Samra Z, Niv Y, et al. Susceptibility-guided vs. empiric retreatment of Helicobacter pylori infection after treatment failure. Dig Dis Sci 2006;51:2316-2321.

53. Cosme A, Lizasoan J, Montes M, et al. Antimicrobial susceptibilityguided therapy versus empirical concomitant therapy for eradication of Helicobacter pylori in a region with high rate of clarithromycin resistance. Helicobacter 2016;21:29-34.

54. Criado RB, Pérez Citores L, Pérez-Millán AG, et al. Prospective comparative study of the treatment of Helicobacter pylori with antibiotic susceptibility testing-guided triple therapy compared to quadruple therapy with Pylera ${ }^{\circ}$. Rev Esp Enferm Dig 2021;113:597-601.

55. Ji CR, Liu J, Li YY, et al. Susceptibility-guided quadruple therapy is not superior to medication history-guided therapy for the rescue treatment of Helicobacter pylori infection: A randomized 
controlled trial. J Dig Dis 2020;21:549-557.

56. Byambajav TO, Bira N, Choijamts G, et al. Initial trials with susceptibility-based and empiric anti-H. pylori therapies in Mongolia. Front Pharmacol 2019;10:394.

57. Ferenc S, Gnus J, Kościelna M, et al. High antibiotic resistance of Helicobacter pylori and its effect on tailored and empiric eradication of the organism in Lower Silesia, Poland. Helicobacter 2017;22.

58. Zhou LY, Zhang JZ, Song Z, et al. Tailored versus triple plus bismuth or concomitant therapy as initial Helicobacter pylori treatment: a randomized trial. Helicobacter 2016;21;91-99.

59. Kim YM, Lee KH, Kim JH, et al. Is only clarithromycin susceptibility important for the successful eradication of Helicobacter pylori? Antibiotics (Basel) 2020;9:589.

60. Lee JW, Kim N, Nam RH, et al. Favorable outcomes of culturebased Helicobacter pylori eradication therapy in a region with high antimicrobial resistance. Helicobacter 2019;24:e12561.

61. Costa S, Soares JB, Gonçalves R. Efficacy and tolerability of culture-guided treatment for Helicobacter pylori infection. Eur $J$ Gastroenterol Hepatol 2017;29:1258-1263.

62. Gisbert JP. Rifabutin for the treatment of Helicobacter pylori infection: a review. Pathogens 2020;10:15.

63. Baylina M, Muñoz N, Sánchez-Delgado J, López-Góngora S, Calvet $\mathrm{X}$, Puig I. Systematic review: would susceptibility-guided treatment achieve acceptable cure rates for second-line Helicobacter pylori therapy as currently practiced? Helicobacter 2019;24:e12584.

64. Gisbert JP. Empirical or susceptibility-guided treatment for Helicobacter pylori infection? A comprehensive review. Therap Adv Gastroenterol 2020;13: 1756284820968736.

65. Graham DY, Lew GM, Ramirez FC, Genta RM, Klein PD, Malaty HM. Short report: a non-metronidazole triple therapy for eradication of Helicobacter pylori infection-tetracycline, amoxicillin, bismuth. Aliment Pharmacol Ther 1993;7:111-113.

66. De Francesco V, Giorgio F, Ierardi E, D’Ercole C, Hassan C, Zullo A. Helicobacter pylori clarithromycin resistance assessment: are gastric antral biopsies sufficient? Gastroenterol Insights 2012;4:7-8.

67. De Francesco V, Zullo A, Manta R, et al. Helicobacter pylori eradication following first-line treatment failure in Europe: what, how and when chose among different standard regimens? A systematic review. Eur J Gastroenterol Hepatol 2021 Mar 19 [Online ahead of print]. doi: 10.1097/MEG.0000000000002100 